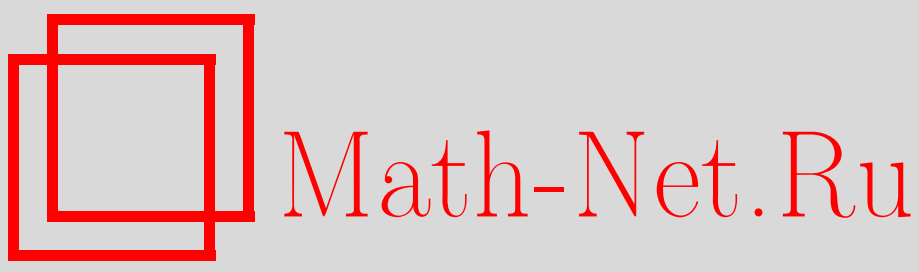

В. А. Мильман, Липшицевы продолжения линейно ограниченных функций, $M a-$ тем. сб., 1998, том 189, номер 8, 67-92

DOI: https://doi.org/10.4213/sm340

Использование Общероссийского математического портала Math-Net.Ru подразумевает, что вы прочитали и согласны с пользовательским соглашением

http://www . mathnet.ru/rus/agreement

Параметры загрузки:

IP: 107.22 .136 .117

26 апреля 2023 г., 18:27:44 
УДК 517.5

\author{
В.А. Мильман
}

\title{
Липшицевы продолжения линейно ограниченных функций
}

\begin{abstract}
Рассматривается задача продолжения действительнозначной функции с подмножества $Y$ метрического пространства $(X, d)$ на все пространство. Известный результат Макшейна позволяет продолжить равномерно непрерьвную функцию с сохранением модуля непрерьвности. Однако при этом остаются без ответа некоторые естественные вопросы. Предлагается новый вид продолжения для широкого класса функций, включающего ограниченные и липшицевы функции. Доказывается ряд полезных в приложениях свойств продолжения, в том числе, сохранение ограничений на приращения значений функции, если ограничения заданы при помощи квазивогнутых мажорант.

Построенное продолжение позволило усилить и обобщить известные результаты в задаче о следах функций с ограниченным градиентом. Продолжение применяется в двух задачах приближения функций. В частности, дано прямое доказательство теоремы о плотности класса $\operatorname{Lip}(X)$ в класcе $\operatorname{lip}(X, \omega)$.
\end{abstract}

Библиографй: 24 названия.

\section{Используемые обозначения и понятия}

В работе используются следуюшие обозначения:

$(X, d)$ - метрическое пространство, $Y$ - замкнутое непустое собственное подмножество $X$;

$x y=d(x, y)$ - расстояние между точками $x, y \in X$;

$d(x, Y)=\inf \{x y: y \in Y\}-$ расстояние от точки $x \in X$ до множества $Y$;

$\left\{y_{i}\right\}_{i=1}^{\infty}-$ последовательность точек из $Y$;

$\operatorname{dom}(h)$ - область определения функции $h$;

$h \mid Y$ - сужение функции $h$ на подмножество $Y$.

Все функции в работе предполагаются действительнозначными. Следуя Н. Бурбаки, функцию $h: \mathbb{R} \rightarrow \mathbb{R}$ будем называть:

возрастающей, если $t_{1}<t_{2} \Rightarrow h\left(t_{1}\right) \leqslant h\left(t_{2}\right)$;

строго возрастающей, если $t_{1}<t_{2} \Rightarrow h\left(t_{1}\right)<h\left(t_{2}\right)$;

убивающей, если $t_{1}<t_{2} \Rightarrow h\left(t_{1}\right) \geqslant h\left(t_{2}\right)$;

строго убивающей, если $t_{1}<t_{2} \Rightarrow h\left(t_{1}\right)>h\left(t_{2}\right)$.

Буквой $\omega$ (возможно с индексами) всегда обозначается функция, действуюшая из $\mathbb{R}^{+}$в $\mathbb{R}^{+}$.

Функция $\omega$ называется полуаддитивной, если

$$
\omega(a+b) \leqslant \omega(a)+\omega(b) \quad \forall a, b \geqslant 0 .
$$


Липшицевой полунормой функции $h: Y \rightarrow \mathbb{R}$ называется величина

$$
\|h\|_{\text {Lip }}=\sup \left\{\frac{|h(x)-h(y)|}{x y}: x, y \in Y, x \neq y\right\} \text {. }
$$

Будем использовать следующие классы функций:

$$
\begin{aligned}
& \operatorname{Lip}(Y)=\left\{h: Y \rightarrow \mathbb{R}:\|h\|_{\text {Lip }}<+\infty\right\}-\text { класс липшицевых функций, } \\
& K \cdot \operatorname{Lip}(Y)=\left\{h: Y \rightarrow \mathbb{R}:\|h\|_{\operatorname{Lip}} \leqslant K\right\} \\
& \operatorname{Lip}(Y, \omega)=\{h: Y \rightarrow \mathbb{R}:|h(x)-h(y)| \leqslant \omega(x y) \forall x, y \in Y\} .
\end{aligned}
$$

Пусть $Z \subset \operatorname{dom}(h), x \in \operatorname{dom}(h)$. Введем обозначение

$$
k[Z] h(x)=\sup \left\{\frac{|h(y)-h(x)|}{x y}: y \in Z, x \neq y\right\}
$$

Число $k[\operatorname{dom}(h)] h(x)$ называется коэффициентом Липиица функции $h$ в точке $x$. Коэффициент Липшица является точной нижней гранью чисел $K$ таких, что выполняется неравенство

$$
|h(y)-h(x)| \leqslant K \cdot x y \quad \forall y \in \operatorname{dom}(h) .
$$

Будем говорить, что для функции $h$ в точке $x$ выполняется (нарушается) условие Липшица, если ее коэффициент Липшица в этой точке конечен (бесконечен).

\section{§1. Введение}

В работе рассматривается задача продолжения функции $f: Y \rightarrow \mathbb{R}$ на все множество $X$. Известно, что функция $f \in K \cdot \operatorname{Lip}(Y)$ может быть продолжена на $X$ с сохранением константы $K$, т.е. сушествует функция $u \in K \cdot \operatorname{Lip}(X)$ такая, что $u \mid Y=f$. Имеются два доказательства этого факта [1]. Первое получено Киршбрауном как частный случай результата о продолжении сжимающих векторнозначных отображений. Доказательство проводится с использованием леммы Цорна по той же схеме, что и традиционное доказательство теоремы Хана-Банаха. Второе доказательство предложил Макшейн [2], указав явную формулу для продолжения:

$$
u(x)=\inf \{f(y)+K x y: y \in Y\}
$$

Оба доказательства неоднократно переоткрывались, например, в [3]-[6]. Продолжение липшицевых функций используется в различных разделах анализа [1], [4]-[13] и в приложениях [14]. Условия непрерывной зависимости продолжений от исходных данных исследованы в [15].

В [2], [3] получено обобщение формулы Макшейна на функции из класса $\operatorname{Lip}(Y, \omega)$, где функция $\omega$ возрастает, полуаддитивна и $\omega(0)=\lim _{t \rightarrow+0} \omega(t)=0$. Если $f \in \operatorname{Lip}(Y, \omega)$, то функция

$$
u(x)=\inf \{f(y)+\omega(x y): y \in Y\}
$$


принадлежит классу $\operatorname{Lip}(X, \omega)$. Функцию $\omega$ с указанньми свойствами называют модулем непрерывности. Поэтому говорят, что продолжение (1.1) сохраняет модуль непрерывности $\omega$.

Принадлежность функции $f$ классу $K \cdot \operatorname{Lip}(Y)$ либо $\operatorname{Lip}(Y, \omega)$ накладывает ограничения на прирашения функции равномерно по всей области определения. Эти ограничения не учитывают того обстоятельства, что возможно различное поведение функции в разных частях области определения. Поэтому упомянутые результаты о продолжении функций не дают ответы на некоторые естественные вопросы. Например, на такой:

всегда ли существует такое продолжение функции, при котором множество точек, где нарушается условие Липшица, одинаково для исходной функиии и для ее продолжения?

ПримеР. Пусть $X=\mathbb{R}^{2}, Y=\{(0, b): b \in \mathbb{R}\}$,

$$
f(0, b)= \begin{cases}0, & \text { если } b \leqslant 0, \\ b^{1 / 2}, & \text { если } b>0 .\end{cases}
$$

Очевидно, что $f \in \operatorname{Lip}\left(Y, t^{1 / 2}\right)$. Применение формулы (1.1) дает продолжение

$$
u(a, b)= \begin{cases}|a|^{1 / 2}, & \text { если } b \leqslant 0, \\ \left(|a|^{2}+|b|^{2}\right)^{1 / 4}, & \text { если } b>0 .\end{cases}
$$

Функция $u$ принадлежит классу $\operatorname{Lip}\left(X, t^{1 / 2}\right)$. Однако для исходной функции $f$ условие Липшица нарушается только в точке $(0,0)$, а для продолжения $u$ это условие нарушается на всей полуоси $\{(0, b): b \leqslant 0\}$.

Возможен также следуюший вопрос:

существует ли продолжение функци, при котором не возрастает коәффициент Липшица в каждой точке множества $Y$ ?

Наконец, возможен вопрос в самой общей форме.

Пусть каждой точке у $\in Y$ поставленъ в соответствие две функции $\omega_{y}^{+}(t)$ и $\omega_{y}^{-}(t)$, ограничивающие приращение значений функции $f$ относительно значения в точке $y$ :

$$
-\omega_{y}^{-}\left(y_{1} y\right) \leqslant f\left(y_{1}\right)-f(y) \leqslant \omega_{y}^{+}\left(y_{1} y\right) \quad \forall y, y_{1} \in Y \text {. }
$$

Существует ли продолжсние функиии, при котором сохраняются ограничения на приращения? То есть существует ли продолжсение и функчии $f$ такое, что

$$
-\omega_{y}^{-}(x y) \leqslant u(x)-u(y) \leqslant \omega_{y}^{+}(x y) \quad \forall y \in Y, \forall x \in X ?
$$

Если продолжсение существует, то будет ли для него выполняться условие Липиица в точках мнохсества $X \backslash Y$ ?

Ответ оказывается положительным для функций, которые мы назвали линейно ограниченными. 
ОПРЕДЕЛЕНИЕ. Функция $f$, определенная на метрическом пространстве с метрикой $d$, называется линейно ограниченной, если существуют $y_{0} \in \operatorname{dom}(f)$, $A, B \in \mathbb{R}^{+}$такие, что

$$
|f(y)| \leqslant A+B d\left(y_{0}, y\right) \quad \forall y \in \operatorname{dom}(f) .
$$

Линейно ограниченные функции могут быть разрьвньми. Ограниченная функция заведомо линейно ограничена. Класс всех линейно ограниченных функций, определенных на $Y$, образует линейное пространство и включает в себя классы $\operatorname{Lip}(Y)$ и $\operatorname{Lip}(Y, \omega)$, где $\omega$ - линейно ограниченная возрастающая функция.

В настоящей работе для линейно ограниченной функции $f$ строится продолжение, даюшее положительньй ответ на сформулированные вопросы. Кроме того, это продолжение обладает рядом полезных свойств, что позволяет в некоторых приложениях усилить результат и/или сократить доказательство.

Работа построена следующим образом. В $§ 2$ приводится неформальная геометрическая конструкция продолжения и доказываются вспомогательные результаты. В $\S 3$ определяется продолжение и устанавливаются его свойства. В $\S 4$ полученные результаты применяются в задаче о следе функции с ограниченным градиентом, рассмотренной в [7] в связи с физическими приложениями. В $\S 5$ продолжение функций применяется в задачах приближения.

Некоторые частные случаи рассматриваемых вопросов ранее излагались в $[16],[17]$.

\section{§2. Вспомогательные результаты}

2.1. Геометрическая конструкция. Приведем неформальную геометрическую конструкцию продолжения линейно ограниченной функции $f: Y \rightarrow \mathbb{R}$. Все приводимые при этом утверждения далее строго доказываются.

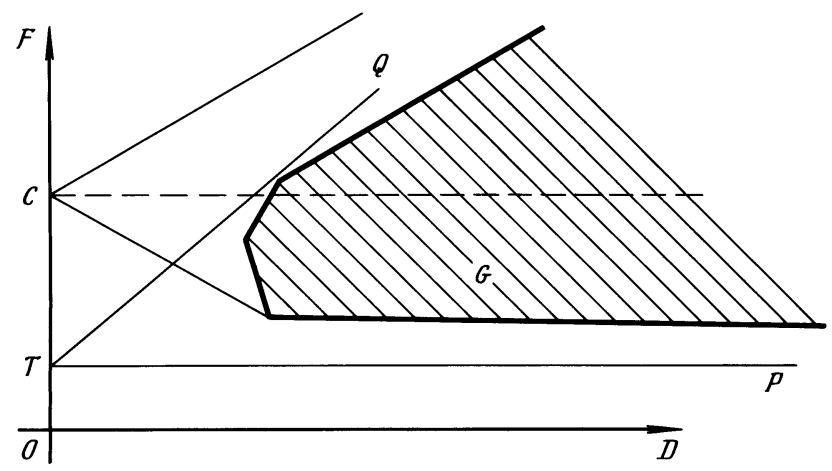

Выберем произвольную точку $x$ из $X \backslash Y$ и определим значение продолжения в этой точке. Для этого на плоскости введем систему координат DOF (см. рис.). По горизонтальной оси $O D$ будем откладьвать расстояния от точки $x$ до точек множества $Y$. По вертикальной оси $O F$ будем откладьвать значения функции $f$. На плоскости определим множество

$$
G=\{(x y, f(y)): y \in Y\}
$$


Отметим, что множество $G$ лежит на положительном расстоянии от оси $O F$, так как множество $Y$ замкнуто и $x \notin Y$.

Возьмем на оси $O F$ произвольную точку $T=(0, t)$. Рассмотрим угол $P T Q$, под которым видно множество $G$ из точки $T$. В силу линейной ограниченности функции $f$ этот угол, исключая точку $T$, лежит строго справа от оси $O F$. Через $(-p(t))$ обозначим тангенс угла наклона прямой $T P$, а через $q(t)$ обозначим тангенс угла наклона прямой $T Q$. Перемешая точку $T$ по оси $O F$, найдем положение, обозначаемое через $C=(0, c)$, когда $p(c)=q(c)$. В этом случае биссектриса угла $P C Q$ расположена горизонтально. Оказывается, возможны два случая:

такое положение точки $C=(0, c)$ единственно;

такие положения заполняют на вертикальной оси конечньй невырожденный отрезок $\left\{(0, c): c_{-} \leqslant c \leqslant c_{+}\right\}$.

В первом случае искомое значение продолжения функции полагаем равным $c$, а во втором - равньм $\left(c_{-}+c_{+}\right) / 2$. Таким образом, продолжение в произвольно выбранной точке определено.

2.2 Вспомогательные функции. Везде далее предполагается, что функция $f: Y \rightarrow \mathbb{R}$ линейно ограничена. Определим вспомогательные функции $p_{f, x}, q_{f, x}$ и исследуем их свойства. Пусть $x \in X \backslash Y, t \in \mathbb{R}$,

$$
\begin{aligned}
& p_{f, x}(t):=\sup \left\{\frac{t-f(y)}{x y}: y \in Y\right\}, \\
& q_{f, x}(t):=\sup \left\{\frac{f(y)-t}{x y}: y \in Y\right\} .
\end{aligned}
$$

Из линейной ограниченности функции $f$ сразу следует конечность функций $p_{f, x}$ и $q_{f, x}$. Следуюшая лемма характеризует зависимость функций $p_{f, x}$ и $q_{f, x}$ от исходной функции $f$.

Лемма 2.1. Пусть $f$ и $g$-линейно ограниченнье функиии, $x \in X \backslash Y$, $a, b \in \mathbb{R}$. Тогдa

1) ecли $f \leqslant g$, mo $p_{f, x} \geqslant p_{g, x}, q_{f, x} \leqslant q_{g, x}$;

2) $p_{f+b, x}(t)=p_{f, x}(t-b), q_{f+b, x}(t)=q_{f, x}(t-b)$;

3) $n$ рu $a \geqslant 0 \quad p_{a f, x}(a t)=a \cdot p_{f, x}(t), q_{a f, x}(a t)=a \cdot q_{f, x}(t)$;

4) при $a<0 \quad p_{a f, x}(a t)=-a \cdot q_{f, x}(t), q_{a f, x}(a t)=-a \cdot p_{f, x}(t)$.

Доказательство непосредственно следует из определений функций $p_{f, x}$ и $q_{f, x}$.

Там, где исходная функция $f$ и/или точка $x$ фиксированы, будем использовать краткие обозначения

$$
p(t)=p_{x}(t)=p_{f, x}(t), \quad q(t)=q_{x}(t)=q_{f, x}(t) .
$$

Далее считаем функцию $f$ фиксированной. Возьмем $x \in X \backslash Y$. В случае, когда множество $Y$ неограничено, определим числа

$$
\begin{aligned}
& p_{0}=\lim _{t \rightarrow+\infty} \sup \left\{-\frac{f(y)}{x y}: y \in Y, x y \geqslant t\right\} ; \\
& q_{0}=\lim _{t \rightarrow+\infty} \sup \left\{\frac{f(y)}{x y}: y \in Y, x y \geqslant t\right\} .
\end{aligned}
$$


Очевидно, что пределы сушествуют, $p_{0}, q_{0}$ не зависят от выбора точки $x$ и выполняются неравенства $p_{0} \leqslant p_{x}(t), q_{0} \leqslant q_{x}(t)$ для любого $t \in \mathbb{R}$. Если множество $Y$ ограничено, положим по определению $p_{0}=q_{0}=-\infty$.

Лемма 2.2. Пусть $x \in X \backslash Y$. Функиия $p=p_{x}$ непрерывна, возрастает, выпукла и

$$
\lim _{t \rightarrow+\infty} p(t)=+\infty
$$

Возможни два случая:

1) существует $t_{p}(x) \in \mathbb{R}$ такое, что $p(t)=p_{0}$ при $t \leqslant t_{p}(x)$ и $p(t)$ строго возрастает при $t>t_{p}(x)$;

2) $p(t)$ строго возрастает на всем $\mathbb{R}$, в этом случае полагаем по определению $t_{p}(x)=-\infty$.

При $t \geqslant t_{p}(x)$ существует последовательность $\left\{y_{i}\right\}_{i=1}^{\infty}$ точек из $Y$ такая, чmo

$$
t=\lim _{i \rightarrow \infty}\left(f\left(y_{i}\right)+p(t) x y_{i}\right) .
$$

При $t>t_{p}(x)$ для каждой такой последовательности выполняются неравенства

$$
\left(D_{+} p(t)\right)^{-1} \leqslant \varliminf_{i \rightarrow \infty} x y_{i} \leqslant \varlimsup_{i \rightarrow \infty} x y_{i} \leqslant\left(D_{-} p(t)\right)^{-1},
$$

где $D_{+}, D_{-}-$правая и левая производнье.

ДокАЗАТЕЛЬСтво. Возрастание функции $p$ очевидно. Функция $p$ определена на $\mathbb{R}$ как поточечная точная верхняя грань множества линейных функций, стремящихся к $+\infty$ при $t \rightarrow+\infty$. Выпуклость и непрерьвность функции $p$ следуют из $[18$, гл. $1, \S 4]$. Оттуда же следует, что возможны два случая:

1 ) сушествует $t_{p}(x) \in \mathbb{R}$ такое, что $p(t)$ постоянна при $t \leqslant t_{p}(x)$ и $p(t)$ строго возрастает при $t>t_{p}(x)$;

2) $p(t)$ строго возрастает на всем $\mathbb{R}$.

Докажем, что $p(t)=p_{0}$ при $t<t_{p}(x)$. Пусть $t<t_{p}(x), \delta>0, t+\delta<t_{p}(x)$. Из определения $p(t)$ следует сушествование последовательности $\left\{y_{i}\right\}_{i=1}^{\infty}$ такой, что вьполняется равенство

$$
p(t)=\lim _{i \rightarrow \infty} \frac{t-f\left(y_{i}\right)}{x y_{i}} .
$$

Из соотношений

$$
p(t+\delta) \geqslant \frac{t+\delta-f\left(y_{i}\right)}{x y_{i}}=\frac{t-f\left(y_{i}\right)}{x y_{i}}+\frac{\delta}{x y_{i}}
$$

и из (2.5) имеем

$$
\lim _{i \rightarrow \infty} x y_{i}=+\infty
$$

Следовательно,

$$
p(t)=\lim _{i \rightarrow \infty} \frac{t-f\left(y_{i}\right)}{x y_{i}} \leqslant \varlimsup_{i \rightarrow \infty}-\frac{f\left(y_{i}\right)}{x y_{i}} \leqslant p_{0} \leqslant p(t),
$$

т.е. $p(t)=p_{0}$. 
Докажем (2.3). Пусть $t \geqslant t_{p}(x)$. Тогда $p(t)<p(t+\delta) \forall \delta>0$. Из определения функции $p$ следует, что

$$
t-f(y)-p(t) x y \leqslant 0 \quad \forall y \in Y
$$

Предположим, что для любой последовательности $\left\{y_{i}\right\}_{i=1}^{\infty}$ равенство (2.3) не выполняется. С учетом последнего неравенства это означает сушествование $\delta>0$ такого, что

$$
t-f(y)-p(t) x y \leqslant-\delta<0 \quad \forall y \in Y
$$

Тогда

$$
\frac{t+\delta-f(y)}{x y} \leqslant p(t) \quad \forall y \in Y,
$$

что противоречит неравенству $p(t+\delta)>p(t)$. Равенство $(2.3)$ доказано.

Докажем первое неравенство в (2.4). Пусть $t>t_{p}(x), \delta>0$ и последовательность $\left\{y_{i}\right\}_{i=1}^{\infty}$ такова, что выполняется (2.3). Из (2.3) и $x y_{i}>d(x, Y)>0$ следует, что $\lim _{i \rightarrow \infty}\left(t-f\left(y_{i}\right)\right) / x y_{i}=p(t)$. Тогда

$$
p(t+\delta) \geqslant \frac{t+\delta-f\left(y_{i}\right)}{x y_{i}}=\frac{t-f\left(y_{i}\right)}{x y_{i}}+\frac{\delta}{x y_{i}}
$$

Переходя к верхнему пределу, получим

$$
p(t+\delta)-p(t) \geqslant \varlimsup_{i \rightarrow \infty} \frac{\delta}{x y_{i}}
$$

откуда следует, что $\left(D_{+} p(t)\right)^{-1} \leqslant \underline{\lim }_{i \rightarrow \infty} x y_{i}$. Вторая часть $(2.4)$ доказьвается аналогично. Лемма доказана.

СледСТвиЕ. Пусть $x \in X \backslash Y$. Функция $q=q_{x}$ непрерьвна, убъвает, выпукла и

$$
\lim _{t \rightarrow-\infty} q(t)=+\infty
$$

Возможны два случая:

1) существует $t_{q}(x) \in \mathbb{R}$ такое, что $q(t)=q_{0}$ при $t \geqslant t_{q}(x)$ и $q(t)$ строго убивает при $t<t_{q}(x)$

2) $q(t)$ строго убшвает на всем $\mathbb{R}$, в этом случае полагаем по определению $t_{q}(x)=+\infty$.

При $t \leqslant t_{q}(x)$ существует последовательность $\left\{y_{i}\right\}_{i=1}^{\infty}$ точек из $Y$ такая, чmo

$$
t=\lim _{i \rightarrow \infty}\left(f\left(y_{i}\right)-q(t) x y_{i}\right) .
$$

При $t<t_{q}(x)$ для каждой такой последовательности выполняются неравенства

$$
\left(D_{-} q(t)\right)^{-1} \leqslant \lim _{i \rightarrow \infty} x y_{i} \leqslant \varlimsup_{i \rightarrow \infty} x y_{i} \leqslant\left(D_{+} q(t)\right)^{-1} .
$$


ДОКАЗАТЕЛЬСТво. Из свойства 4) в лемме 2.1 следует, что

$$
q_{-f, x}(-t)=p_{f, x}(t) .
$$

Поэтому свойства функции $q_{f, x}$ получаются из леммы 2.2 как следствия свойств функции $p_{f, x}$. Следствие доказано.

Рассмотрим уравнение

$$
p_{f, x}(t)=q_{f, x}(t),
$$

играющее центральную роль при построении продолжения. Исследуем структуру множества его решений при фиксированном $x \in X \backslash Y$.

Лемма 2.3. Множество решений уравнения (2.6) представляет собой конечный отрезок $\left[c_{-}, c_{+}\right]$, который может быть вырожденным, т.е. возможен случай $c_{+}=c_{-}$.

Если число с является решением уравнения (2.6) и $c_{1} \in \mathbb{R}$, то

$$
0 \leqslant p(c)=q(c)=\sup \left\{\frac{|f(y)-c|}{x y}: y \in Y\right\} \leqslant \sup \left\{\frac{\left|f(y)-c_{1}\right|}{x y}: y \in Y\right\} .
$$

ДокаЗАТЕЛЬСтво. Функция $p(t)-q(t)$ непрерывна и возрастает. В силу монотонности функций $p$ и $q$

$$
\begin{aligned}
& \lim _{t \rightarrow-\infty}(p(t)-q(t)) \leqslant p(0)-\lim _{t \rightarrow-\infty} q(t)=-\infty, \\
& \lim _{t \rightarrow+\infty}(p(t)-q(t)) \geqslant \lim _{t \rightarrow+\infty} p(t)-q(0)=+\infty .
\end{aligned}
$$

Значит, множество решений уравнения (2.6) непусто, ограничено, замкнуто и связно, т.е. представляет собой либо конечный невырожденный отрезок, либо единственное число.

Предположим, что $p(c)=q(c)<0$. Возьмем произвольное $y \in Y$. Тогда $(c-f(y)) / x y \leqslant p(c)<0$ и, следовательно, $q(c) \geqslant(f(y)-c) / x y>0$. Полученное противоречие показывает, что $p(c)=q(c) \geqslant 0$.

Докажем второе неравенство в (2.7). Очевидно, что

$$
\sup \left\{\frac{|f(y)-t|}{x y}: y \in Y\right\}=\max \{p(t) ; q(t)\} .
$$

Если $c_{1} \geqslant c$, то $p\left(c_{1}\right) \geqslant p(c)=q(c)$. Если $c_{1}<c$, то $q\left(c_{1}\right) \geqslant q(c)=p(c)$. Поэтому

$$
\begin{aligned}
\sup \left\{\frac{\left|f(y)-c_{1}\right|}{x y}: y \in Y\right\} & =\max \left\{p\left(c_{1}\right) ; q\left(c_{1}\right)\right\} \\
& \geqslant p(c)=q(c)=\sup \left\{\frac{|f(y)-c|}{x y}: y \in Y\right\} .
\end{aligned}
$$

Лемма доказана.

Введем обозначение

$$
L(x)=p_{f, x}(c)=q_{f, x}(c),
$$


где $c$ - любой корень уравнения (2.6). Непосредственно из определений функций $p$ и $q$ следуют два свойства функции $L(x)$ :

1) выполняются неравенства

$$
f(y)-L(x) x y \leqslant c \leqslant f(y)+L(x) x y \quad \forall y \in Y ;
$$

2) для любого $\varepsilon>0$ сушествуют $y_{1}, y_{2} \in Y$ такие, что

$$
\frac{c-f\left(y_{1}\right)}{x y_{1}} \geqslant L(x)-\varepsilon, \quad \frac{f\left(y_{2}\right)-c}{x y_{2}} \geqslant L(x)-\varepsilon .
$$

Лемма 2.4. Пусть $x \in X \backslash Y$. Справедливо равенство

$$
L(x)=\sup \left\{\frac{f\left(y_{1}\right)-f\left(y_{2}\right)}{x y_{1}+x y_{2}}: y_{1}, y_{2} \in Y\right\} .
$$

Функция $L(x)$ непрерывна в точке $x$ и ограничена в некоторой окрестности этой точки.

ДокАЗАТЕЛЬСтво. Пусть $y_{1}, y_{2} \in Y$. Из (2.8) следуют неравенства

$$
f\left(y_{1}\right)-c \leqslant L(x) x y_{1}, \quad c-f\left(y_{2}\right) \leqslant L(x) x y_{2} .
$$

Складывая эти неравенства, получаем

$$
f\left(y_{1}\right)-f\left(y_{2}\right) \leqslant L(x)\left(x y_{1}+x y_{2}\right) .
$$

Возьмем $\varepsilon>0$. Выбрав точки $y_{1}, y_{2} \in Y$, удовлетворяющие условию (2.9), получим неравенства

$$
f\left(y_{1}\right)-c \geqslant(L(x)-\varepsilon) x y_{1}, \quad c-f\left(y_{2}\right) \geqslant(L(x)-\varepsilon) x y_{2} .
$$

Складывая эти неравенства, получаем

$$
f\left(y_{1}\right)-f\left(y_{2}\right) \geqslant(L(x)-\varepsilon)\left(x y_{1}+x y_{2}\right) .
$$

Учитывая, что $\varepsilon$ может быть выбрано произвольно малым, из (2.10) и (2.11) получаем требуемое равенство.

Докажем непрерывность $L(x)$. Возьмем точку $x_{1}$ так близко к $x$, чтобы выполнялось $x x_{1} \leqslant d(x, Y) / 2$ и, следовательно,

$$
d\left(x_{1}, Y\right) \geqslant d(x, Y)-x x_{1} \geqslant \frac{d(x, Y)}{2} .
$$

Справедливы неравенства

$$
\begin{aligned}
\left|L(x)-L\left(x_{1}\right)\right| & \leqslant \sup \left\{\left(f\left(y_{1}\right)-f\left(y_{2}\right)\right)\left(\frac{1}{x y_{1}+x y_{2}}-\frac{1}{x_{1} y_{1}+x_{1} y_{2}}\right): y_{1}, y_{2} \in Y\right\} \\
& =\sup \left\{\frac{f\left(y_{1}\right)-f\left(y_{2}\right)}{x y_{1}+x y_{2}} \cdot \frac{x_{1} y_{1}+x_{1} y_{2}-x y_{1}-x y_{2}}{x_{1} y_{1}+x_{1} y_{2}}: y_{1}, y_{2} \in Y\right\} \\
& \leqslant L(x) \frac{x x_{1}}{d\left(x_{1}, Y\right)} \leqslant L(x) \frac{2 x x_{1}}{d(x, Y)},
\end{aligned}
$$

откуда следует непрерывность $L(x)$ в точке $x$ и ограниченность $L(x)$ в некоторой окрестности этой точки. Лемма доказана.

Рассмотрим зависимость множества решений уравнения (2.6) от точки $x$. Покажем, что функции $c_{-}(x), c_{+}(x)$ удовлетворяют условию Липшица на $X \backslash Y$ и их коэффициент Липшица в точке $x$ не превосходит $L(x)$. 
Лемма 2.5. Если $x, z \in X \backslash Y, m o$

$$
\begin{aligned}
& \left|c_{+}(x)-c_{+}(z)\right| \leqslant \min \{L(x) ; L(z)\} x z, \\
& \left|c_{-}(x)-c_{-}(z)\right| \leqslant \min \{L(x) ; L(z)\} x z .
\end{aligned}
$$

ДокАЗАТЕЛЬСтво. Докажем только первое неравенство, так как второе доказывается аналогично. Для определенности будем считать, что $L(x) \geqslant L(z)$. Возможны следующие случаи:

1) $L(x)>L(z)$;

1.1) $c_{+}(x) \geqslant c_{+}(z)$

1.2) $c_{+}(x)<c_{+}(z)$

2) $L(x)=L(z)$

2.1) $L(x)>p_{0}, L(x)>q_{0}$;

2.2) $L(x)=p_{0}=q_{0}$

2.3) $L(x)=q_{0}>p_{0}$

2.4) $L(x)=p_{0}>q_{0}$.

В случаях 1.2), 2.1), 2.2), 2.3) из леммы 2.2 следует сушествование последовательности $\left\{y_{i}\right\}_{i=1}^{\infty}$ такой, что

$$
c_{+}(x)=f\left(y_{i}\right)+L(x) x y_{i}+a_{i},
$$

где $\lim _{i \rightarrow \infty} a_{i}=0$. Из (2.8) следует неравенство

$$
c_{+}(z) \leqslant f\left(y_{i}\right)+L(z) z y_{i} .
$$

Из (2.12) и (2.13) получаем

$$
c_{+}(z)-c_{+}(x) \leqslant-L(x) x y_{i}+L(z) z y_{i}-a_{i} \leqslant L(z)\left(z y_{i}-x y_{i}\right)-a_{i} \leqslant L(z) x z-a_{i} .
$$

Переходя к пределу при $i \rightarrow \infty$, получаем

$$
c_{+}(z)-c_{+}(x) \leqslant L(z) x z .
$$

Заметим, что в случае 2) в силу симметрии между $x$ и $z$ можно считать, что $c_{+}(x) \leqslant c_{+}(z)$. Таким образом, в рассмотренных случаях требуемое неравенство доказано.

В случаях 1.1), 2.4) согласно следствию леммы 2.2 сушествует последовательность $\left\{y_{i}\right\}_{i=1}^{\infty}$ такая, что

$$
c_{+}(x)=f\left(y_{i}\right)-L(x) x y_{i}+a_{i},
$$

где $\lim _{i \rightarrow \infty} a_{i}=0$. Из (2.8) следует неравенство

$$
c_{+}(z) \geqslant f\left(y_{i}\right)-L(z) z y_{i} .
$$

Из (2.14) и (2.15) получаем

$c_{+}(x)-c_{+}(z) \leqslant-L(x) x y_{i}+L(z) z y_{i}-a_{i} \leqslant L(z)\left(z y_{i}-x y_{i}\right)-a_{i} \leqslant L(z) x z-a_{i}$. 
Переходя к пределу при $i \rightarrow \infty$, получаем

$$
c_{+}(x)-c_{+}(z) \leqslant L(z) x z .
$$

Заметим, что в случае 2) в силу симметрии между $x$ и $z$ можно считать, что $c_{+}(x) \geqslant c_{+}(z)$. Таким образом, в случаях 1.1$\left.), 2.4\right)$ требуемое неравенство доказано. Лемма доказана.

Отметим, что множество решений уравнения (2.6) может быть невырожденньп отрезком только в случае 2.2). Во всех остальных случаях решение единственно, т.е. $c_{-}(x)=c_{+}(x)$. В частности, если множество $Y$ ограничено, то решение единственно.

Следующая лемма позволяет проследить аналогию между функциями $c_{-}(x)$, $c_{+}(x)$ и продолжением (1.1).

Лемма 2.6. Пусть $x \in X \backslash Y$. Если $p_{0}>q_{0}$, mo

$$
c_{+}(x)=c_{-}(x)=\sup \{f(y)-L(x) x y: y \in Y\} .
$$

Ecлu $p_{0}<q_{0}$, mo

$$
c_{+}(x)=c_{-}(x)=\inf \{f(y)+L(x) x y: y \in Y\} \text {. }
$$

Eсли $p_{0}=q_{0}$, mo

$$
\begin{aligned}
& c_{+}(x)=\inf \{f(y)+L(x) x y: y \in Y\} \\
& c_{-}(x)=\sup \{f(y)-L(x) x y: y \in Y\} .
\end{aligned}
$$

ДокАЗАТЕЛЬство. Докажем равенство (2.16). Из (2.8) следует, что

$$
c_{+}(x) \geqslant c_{-}(x) \geqslant \sup \{f(y)-L(x) x y: y \in Y\} .
$$

Далее воспользуемся следствием леммы 2.2. Из неравенства $q_{x}\left(c_{+}(x)\right) \geqslant p_{0}>q_{0}$ имеем $c_{+}(x)<t_{q}(x)$. Из строгого убьвания $q_{x}(t)$ при $t<t_{q}(x)$ имеем $c_{+}(x)=c_{-}(x)$. Из сушествования последовательности $\left\{y_{i}\right\}_{i=1}^{\infty}$ такой, что

$$
c_{+}(x)=c_{-}(x)=\lim _{i \rightarrow \infty}\left(f\left(y_{i}\right)-L(x) x y_{i}\right)
$$

следует неравенство $c_{-}(x) \leqslant \sup \{f(y)-L(x) x y: y \in Y\}$. Равенство (2.16) доказано. Остальные равенства доказываются аналогично. Лемма доказана. 


\section{§ 3. Продолжение с минимальным коэффициентом Липшица}

\section{1. Определение и основные свойства.}

ОПРЕДЕЛЕниЕ. Продолжение линейно ограниченной функции $f: Y \rightarrow \mathbb{R}$, задаваемое формулой

$$
u(x)= \begin{cases}f(x), & \text { если } x \in Y, \\ \frac{c_{-}(x)+c_{+}(x)}{2}, & \text { если } x \in X \backslash Y,\end{cases}
$$

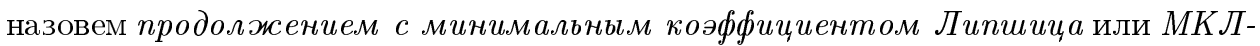
продолжсением. Оператор, ставящий в соответствие функции $f$ ее МКЛ-продолжение $u$, обозначим через $E_{k}$ :

$$
u=E_{k} f
$$

Оператор $E_{k}$ нелинейньй, что видно из следуюшего примера.

Пример. Пусть $X=[0,2], x y=|x-y|, Y=\{0,1,2\}$. На $Y$ заданы две функции $f$ и $f_{1}: f(0)=0, f(1)=0, f(2)=1, f_{1}(0)=1, f_{1}(1)=0, f_{1}(2)=0$. Тогда

$$
\begin{gathered}
E_{k} f(x)= \begin{cases}\frac{x}{2}, & \text { если } 0 \leqslant x<\frac{1}{2}, \\
\frac{1-x}{3-2 x}, & \text { если } \frac{1}{2} \leqslant x<1, \\
x-1, & \text { если } 1 \leqslant x \leqslant 2,\end{cases} \\
E_{k} f_{1}(x)= \begin{cases}\frac{1-x,}{2-1}, & \text { если } 0 \leqslant x<1, \\
\frac{x-1}{2 x-1} & \text { если } 1 \leqslant x<\frac{3}{2}, \\
\frac{1-x}{2}, & \text { если } \frac{3}{2} \leqslant x \leqslant 2 .\end{cases}
\end{gathered}
$$

Непосредственно проверяется, что $E_{k}\left(f+f_{1}\right) \neq E_{k} f+E_{k} f_{1}$.

Tеорема 3.1. Пусть $f, f_{1}$ - линейно ограниченные функиии, $a, b \in \mathbb{R}$, $u=E_{k} f, u_{1}=E_{k} f_{1}$. Оператор $E_{k}$ обладает следующими свойствами:

1) сохраняет верхнюю и нижнюю грани значений функции

$$
\inf \{f(y): y \in Y\} \leqslant u(x) \leqslant \sup \{f(y): y \in Y\} ;
$$

2) является монотонним

$$
f \leqslant f_{1} \Rightarrow u \leqslant u_{1}
$$

3) однороден и аддитивен по отношению к константам

$$
E_{k}(a f+b)=a E_{k} f+b
$$

4) непрерывен относительно равномерной нормы

$$
\sup \left\{\left|f(y)-f_{1}(y)\right|: y \in Y\right\} \leqslant \varepsilon \Rightarrow \sup \left\{\left|u(x)-u_{1}(x)\right|: x \in X\right\} \leqslant \varepsilon .
$$


ДокАЗАТЕЛЬСтво. Докажем только второе неравенство в свойстве 1), так как первое доказывается аналогично. Пусть $x \in X \backslash Y, y_{1} \in Y$. Предположим, что $u(x)>\sup \{f(y): y \in Y\}$. Тогда

$$
\begin{aligned}
& p_{f, x}(u(x))=\sup \left\{\frac{u(x)-f(y)}{x y}: y \in Y\right\} \geqslant \frac{u(x)-f\left(y_{1}\right)}{x y_{1}}>0, \\
& q_{f, x}(u(x))=\sup \left\{\frac{f(y)-u(x)}{x y}: y \in Y\right\} \leqslant 0 .
\end{aligned}
$$

Следовательно, $u(x)$ не является решением уравнения (2.6), что противоречит определению $u(x)$.

Свойства 2) и 3) получаются очевидным образом из леммы 2.1 .

Докажем свойство 4). Пусть

$$
-\varepsilon \leqslant f(y)-f_{1}(y) \leqslant \varepsilon \quad \forall y \in Y .
$$

Тогда в силу свойств 2) и 3 )

$$
\begin{gathered}
u_{1}(x)-\varepsilon \leqslant u(x) \leqslant u_{1}(x)+\varepsilon, \\
-\varepsilon \leqslant u(x)-u_{1}(x) \leqslant \varepsilon .
\end{gathered}
$$

Свойство 4) и теорема доказаны.

Лемма 3.1. Пусть $u=E_{k} f, x, z \in X \backslash Y$. Тогдa

$$
|u(x)-u(z)| \leqslant \min \{L(x) ; L(z)\} x z .
$$

ДокАЗАТЕЛЬСтво. Неравенство следует из леммы 2.5 .

Следующая теорема устанавливает экстремальное свойство МКЛ-продолжения.

ТЕОРема 3.2. МКЛ-продолжение $u=E_{k} f$ имеет в каждой точке $x \in X \backslash Y$ конечныцй, непрерывно зависящий от $x$ и ограниченный в некоторой окрестности х коэффициент Липшица

$$
k[X] u(x)=L(x)=\sup \left\{\frac{f\left(y_{1}\right)-f\left(y_{2}\right)}{x y_{1}+x y_{2}}: y_{1}, y_{2} \in Y\right\} .
$$

Справедливо неравенство

$$
k[X] u(x) \leqslant k[X] v(x),
$$

где $v$ - любое другое продолэсение функиии $f$. 
ДокАЗАТЕЛЬСтво. Из определения величин $k[X] u(x)$ и $L(x)$ следует, что

$$
k[X] u(x)=\max \{k[Y] u(x) ; k[X \backslash Y] u(x)\}=\max \{L(x) ; k[X \backslash Y] u(x)\} .
$$

Из леммы 3.1 следует, что $k[X \backslash Y] u(x) \leqslant L(x)$. Значит, $k[X] u(x)=L(x)$. Из этого равенства и леммы 2.3 получается неравенство (3.1). Непрерьвность $L(x)$, ограниченность $L(x)$ в некоторой окрестности $x$ и формула для $L(x)$ получены в лемме 2.4. Теорема доказана.

Таким образом, МКЛ-продолжение имеет в каждой точке множества $X \backslash Y$ коэффициент Липшица, минимальный среди совокупности всех возможных продолжений. Это оправдьвает название введенного продолжения.

3.2. Сохранение ограничений на приращения. Для ограничения приращений значений функций будем использовать квазивогнутые функции. Эти функции в таком качестве используются в [15], [19], [20].

ОПРЕДЕЛЕНИЕ. Функция $\omega$ называется квазивогнутой, если она удовлетворяет следующим условиям:

1) $\omega(0)=0$;

2) $\omega(t)$ возрастает;

3) $\omega(t) / t$ убывает.

Термин введен в $[19$, гл. $2, \S 1]$ и обусловлен тем, что вогнутая возрастающая неотрицательная функция квазивогнута. Если не указано иное, не предполагается, что $\omega(t)$ непрерывна в нуле. Это позволяет мажорировать приращения значений функции при помощи квазивогнутых функций как в точках непрерывности, так и в точках разрьва. Покажем что МКЛ-продолжение сохраняет ограничения на прирашения значений функции, заданные с помощью квазивогнутых мажорант.

ТЕОРема 3.3. Пусть $x \in X, u-M K Л$-продолжение функции $f$, а функиии $\omega_{x}^{+}, \omega_{x}^{-}$квазивогнуты. Если выполняется неравенство

$$
f(y)-u(x) \leqslant \omega_{x}^{+}(y x) \quad \forall y \in Y,
$$

mo

$$
u\left(x_{1}\right)-u(x) \leqslant \omega_{x}^{+}\left(x_{1} x\right) \quad \forall x_{1} \in X
$$

Если выполняется неравенство

$$
u(x)-f(y) \leqslant \omega_{x}^{-}(y x) \quad \forall y \in Y,
$$

mo

$$
u(x)-u\left(x_{1}\right) \leqslant \omega_{x}^{-}\left(x_{1} x\right) \quad \forall x_{1} \in X .
$$


ДокАЗАТЕЛЬСтво. Пусть $x_{1} \in X \backslash Y, x_{1} \neq x$ и $\varepsilon>0$. В соответствии с (2.9) выберем $y$ так, что

$$
L\left(x_{1}\right)-\varepsilon \leqslant \frac{f(y)-u\left(x_{1}\right)}{x_{1} y} .
$$

Воспользовавшись леммой 3.1, получаем

$$
\begin{gathered}
\frac{u\left(x_{1}\right)-u(x)}{x x_{1}}-\varepsilon \leqslant L\left(x_{1}\right)-\varepsilon \leqslant \frac{f(y)-u\left(x_{1}\right)}{x_{1} y}, \\
\left(u\left(x_{1}\right)-u(x)\right) x_{1} y-\varepsilon \cdot x_{1} y \cdot x x_{1} \leqslant\left(f(y)-u\left(x_{1}\right)\right) x x_{1} .
\end{gathered}
$$

Прибавив к обеим частям неравенства величину $\left(u\left(x_{1}\right)-u(x)\right) x x_{1}$, получаем

$$
\begin{gathered}
\left(u\left(x_{1}\right)-u(x)\right)\left(x_{1} y+x x_{1}\right)-\varepsilon \cdot x_{1} y \cdot x x_{1} \leqslant(f(y)-u(x)) x x_{1}, \\
u\left(x_{1}\right)-u(x) \leqslant \frac{(f(y)-u(x)) x x_{1}}{x_{1} y+x x_{1}}+\varepsilon \cdot x x_{1} .
\end{gathered}
$$

Воспользовавшись неравенством (3.2) и возрастанием функции $\omega_{x}^{+}$, получаем

$$
f(y)-u(x) \leqslant \omega_{x}^{+}(x y) \leqslant \omega_{x}^{+}\left(x_{1} y+x x_{1}\right) .
$$

Из (3.4), последнего неравенства и убывания функции $\omega_{x}^{+}(t) / t$ следует

$$
u\left(x_{1}\right)-u(x) \leqslant \frac{\omega_{x}^{+}\left(x_{1} y+x x_{1}\right) x x_{1}}{x_{1} y+x x_{1}}+\varepsilon \cdot x x_{1} \leqslant \omega_{x}^{+}\left(x x_{1}\right)+\varepsilon x x_{1} .
$$

Так как $\varepsilon$ может быть выбрано произвольно мальп, первое неравенство доказано. Второе доказывается аналогично. Теорема доказана.

СлЕДСТвИЕ. Если в точке $y \in Y$ для функции $f$ выполнено условие Липшица, то это условие в этой же точке выполнено для МКЛ-продолэсения $и$, кроме того,

$$
k[Y] f(y)=k[X] u(y)
$$

ДоКАЗАТЕЛЬСтво. Утверждение следует из теоремы 3.3 при

$$
\omega_{x}^{+}(t)=\omega_{x}^{-}(t)=k[Y] f(y) \cdot t
$$

Рассмотрим продолжение функций из класса $\operatorname{Lip}(Y, \omega)$.

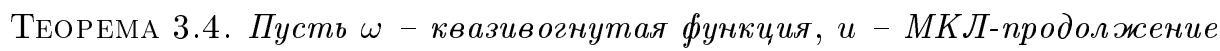
функиии $f \in \operatorname{Lip}(Y, \omega)$. Тогда $u \in \operatorname{Lip}(X, \omega)$ и для всех $x, x_{1} \in X, x_{1} \neq x$, выполняется неравенство

$$
\left|u\left(x_{1}\right)-u(x)\right| \leqslant \frac{\omega\left(d(x, Y)+x x_{1}\right)}{d(x, Y)+x x_{1}} x x_{1} .
$$


ДоказАТЕльство. Из убывания функции $\omega(t) / t$ следует, что правая часть неравенства не превосходит $\omega\left(x x_{1}\right)$. Поэтому выполнение неравенства гарантирует, что $u \in \operatorname{Lip}(X, \omega)$.

Докажем неравенство

$$
f\left(y_{1}\right)-u(x) \leqslant \frac{\omega\left(d(x, Y)+x y_{1}\right)}{d(x, Y)+x y_{1}} x y_{1}
$$

при $y_{1} \in Y, x \in X \backslash Y$. В соответствии с (2.9) выберем $y$ так, что

$$
L(x)-\varepsilon \leqslant \frac{u(x)-f(y)}{x y} .
$$

Воспользовавшись (2.8), получаем

$$
\begin{gathered}
\frac{f\left(y_{1}\right)-u(x)}{x y_{1}}-\varepsilon \leqslant L(x)-\varepsilon \leqslant \frac{u(x)-f(y)}{x y}, \\
\left(f\left(y_{1}\right)-u(x)\right) x y-\varepsilon \cdot x y_{1} \cdot x y \leqslant(u(x)-f(y)) x y_{1} .
\end{gathered}
$$

Прибавив к обеим частям неравенства величину $\left(f\left(y_{1}\right)-u(x)\right) x y_{1}$, получаем

$$
\begin{gathered}
\left(f\left(y_{1}\right)-u(x)\right)\left(x y_{1}+x y\right)-\varepsilon \cdot x y_{1} \cdot x y \leqslant\left(f\left(y_{1}\right)-f(y)\right) x y_{1}, \\
f\left(y_{1}\right)-u(x) \leqslant \frac{\left(f\left(y_{1}\right)-f(y)\right) x y_{1}}{x y+x y_{1}}+\varepsilon \cdot x y_{1} .
\end{gathered}
$$

Воспользовавшись неравенством $f\left(y_{1}\right)-f(y) \leqslant \omega\left(y y_{1}\right)$ и возрастанием функции $\omega$, получаем

$$
f\left(y_{1}\right)-f(y) \leqslant \omega\left(y y_{1}\right) \leqslant \omega\left(x y+x y_{1}\right) .
$$

Из (3.6), последнего неравенства и убывания функции $\omega(t) / t$ следует

$$
f\left(y_{1}\right)-u(x) \leqslant \frac{\omega\left(x y+x y_{1}\right) x y_{1}}{x y+x y_{1}}+\varepsilon \cdot x y_{1} \leqslant \omega \frac{\left(d(x, Y)+x y_{1}\right) x y_{1}}{d(x, Y)+x y_{1}}+\varepsilon \cdot x y_{1} .
$$

Так как $\varepsilon$ может быть выбрано произвольно малым, неравенство (3.5) доказано.

Рассмотрим функцию

$$
\omega_{x}^{+}(t)=\frac{\omega(d(x, Y)+t)}{d(x, Y)+t} t .
$$

Убывание функции $\omega_{x}^{+}(t) / t$ очевидно. Функция $\omega_{x}^{+}$является произведением возрастающих при $t>0$ функций $\omega(d(x, Y)+t)$ и $t /(d(x, Y)+t)$. Следовательно, $\omega_{x}^{+}$ возрастает и является квазивогнутой. Из теоремы 3.3 и неравенства (3.5) следует неравенство

$$
u\left(x_{1}\right)-u(x) \leqslant \frac{\omega\left(d(x, Y)+x x_{1}\right)}{d(x, Y)+x x_{1}} x x_{1} \quad \forall x_{1} \in X, x_{1} \neq x .
$$

Аналогично получается такая же оценка сверху для $u(x)-u\left(x_{1}\right)$ Теорема доказана. 
При продолжении функций из класса $\operatorname{Lip}(Y, \omega)$ между оператором MКЛ-продолжения и оператором, задаваемым формулой (1.1), имеется сушественное различие. Оператор (1.1) определен для конкретного класса $\operatorname{Lip}(Y, \omega)$ и зависит от функции $\omega$. Оператор МКЛ-продолжения единьм образом обеспечивает продолжение функции из любого класса $\operatorname{Lip}(Y, \omega)$, если $\omega$ - квазивогнутая функция.

В формуле (1.1) требуется полуаддитивность функции $\omega$, что несколько слабее требования убывания функции $\omega(t) / t$. Однако последнее различие, как правило, не существенно по следуюшей причине. Если функция $\omega$ возрастает, полуаддитивна, непрерывна в нуле и $\omega(0)=0$, то, как показано в [20, п. 3.2], сушествует квазивогнутая функция $\omega_{1}$ такая, что

$$
\frac{1}{2} \omega(t) \leqslant \omega_{1}(t) \leqslant \omega(t) \quad \forall t \geqslant 0
$$

Рассмотрим вопрос о построении квазивогнутых функций $\omega_{x}^{+}, \omega_{x}^{-}$, обеспечиваюших выполнение условий (3.2) и (3.3). Если $\omega$ - неотрицательная, возрастаюшая, линейно ограниченная функция, то через $(\omega)^{*}$ будем обозначать функцию, определенную формулой

$$
(\omega)^{*}(t)= \begin{cases}0, & \text { если } t=0, \\ t \cdot \sup \left\{\frac{\omega(s)}{s}: s \geqslant t\right\}, & \text { если } t>0 .\end{cases}
$$

Построение функций $\omega_{x}^{+}, \omega_{x}^{-}$основано на следующей теореме.

ТЕОРема 3.5. Пусть $\omega$ - линейно ограниченная, неотрицательная, возрастающая функиия. Тогда $(\omega)^{*}$ обладает следующими свойствами.

1) Функиия $(\omega)^{*}$ является наименьшей квазивогнутой мажорантой для $\omega$, т.е. $(\omega)^{*}$ - квазивогнута и $\omega_{1}(t) \geqslant(\omega)^{*}(t) \geqslant \omega(t)$, где $\omega_{1}-$ любая квазивогнутая функция, превосходящая $\omega$.

2) $E с л и \sup \{\omega(s) / s: s>0\}=+\infty$, то для некоторого $t_{0}>0$ выполняется следующее: для любого $t \in\left(0, t_{0}\right]$ существует $s_{t} \geqslant t$ такое, что

$$
\frac{(\omega)^{*}(t)}{t}=\frac{\omega\left(s_{t}+0\right)}{s_{t}}, \quad(\omega)^{*}\left(s_{t}\right)=\omega\left(s_{t}+0\right), \quad \lim _{t \rightarrow+0} s_{t}=0 .
$$

ДоКАЗАТЕЛЬСТво. Определим функции

$$
\begin{gathered}
r(t)=\sup \left\{\frac{\omega(s)}{s}: s \geqslant t\right\}, \quad t>0, \\
r_{2}\left(t_{1}, t_{2}\right)=\sup \left\{\frac{\omega(s)}{s}: t_{1} \leqslant s \leqslant t_{2}\right\}, \quad t_{2} \geqslant t_{1}>0 .
\end{gathered}
$$

Конечность $r(t), r_{2}\left(t_{1}, t_{2}\right)$ следует из линейной ограниченности $\omega$. Очевидно, что $r$ убывает и что выполняется неравенство $r_{2}\left(t_{1}, t_{2}\right) \leqslant \omega\left(t_{2}\right) / t_{1}$.

В свойстве 1) не является очевидньт только возрастание функции $(\omega)^{*}(t)$ при $t>0$. Пусть $t_{2}>t_{1}>0$. Если $r\left(t_{2}\right)=r\left(t_{1}\right)$, то справедливость неравенства 
$(\omega)^{*}\left(t_{2}\right) \geqslant(\omega)^{*}\left(t_{1}\right)$ очевидна. Если $r\left(t_{2}\right)<r\left(t_{1}\right)$, то $r\left(t_{1}\right)=r_{2}\left(t_{1}, t_{2}\right)$. Следовательно,

$$
(\omega)^{*}\left(t_{1}\right)=t_{1} \cdot r_{2}\left(t_{1}, t_{2}\right) \leqslant t_{1}\left(\frac{\omega\left(t_{2}\right)}{t_{1}}\right)=\omega\left(t_{2}\right) \leqslant(\omega)^{*}\left(t_{2}\right) .
$$

Докажем свойство 2). Очевидно, что если $\sup \{\omega(s) / s: s>0\}=+\infty$, то $r(t) \rightarrow+\infty$ при $t \rightarrow+0$. Так как $r(t)$ убывает и $r(t) \geqslant 0$, то существует предел $r(t)$ при $t \rightarrow+\infty$. Возьмем $t_{0}>0$ так, чтобы

$$
r\left(t_{0}\right) \geqslant 1+\lim _{t \rightarrow+\infty} r(t) .
$$

Пусть $0<t<t_{0}$. Возьмем последовательность $\left\{s_{i}\right\}_{i=1}^{\infty}$ такую, что $s_{i} \geqslant t$ и $\lim _{i \rightarrow \infty} \omega\left(s_{i}\right) / s_{i}=r(t)$. Последовательность ограничена. В противном случае из нее можно выбрать стремящуюся к $+\infty$ подпоследовательность $\left\{s_{i}^{*}\right\}$, что приводит к противоречию:

$$
r(t)=\lim _{i \rightarrow \infty} \frac{\omega\left(s_{i}^{*}\right)}{s_{i}^{*}} \leqslant \lim _{i \rightarrow \infty} r\left(s_{i}^{*}\right)<r\left(t_{0}\right) \leqslant r(t) .
$$

Возьмем $s_{t}=\underline{\lim }_{i \rightarrow \infty} s_{i}$. Тогда $s_{t} \geqslant t$ и

$$
r(t)=\lim _{i \rightarrow \infty} \frac{\omega\left(s_{i}\right)}{s_{i}} \leqslant \frac{\omega\left(s_{t}+0\right)}{s_{t}} .
$$

С другой стороны, из определения $r(t)$ следует, что $r(t) \geqslant \omega\left(s_{t}+0\right) / s_{t}$. Значит, $r(t)=\omega\left(s_{t}+0\right) / s_{t}$.

Очевидным образом получаются неравенства

$$
\frac{\omega\left(s_{t}+0\right)}{s_{t}} \leqslant r\left(s_{t}\right) \leqslant r(t)=\frac{\omega\left(s_{t}+0\right)}{s_{t}} .
$$

Следовательно, $r\left(s_{t}\right)=r(t)=\omega\left(s_{t}+0\right) / s_{t}$ и

$$
(\omega)^{*}\left(s_{t}\right)=s_{t} \cdot r\left(s_{t}\right)=\omega\left(s_{t}+0\right) .
$$

Учитывая, что $\lim _{t \rightarrow+0} r\left(s_{t}\right)=\lim _{t \rightarrow+0} r(t)=+\infty$, получаем $\lim _{t \rightarrow+0} s_{t}=0$. Теорема доказана.

Из теоремы следует равенство

$$
\lim _{t \rightarrow+0} \omega(t)=\lim _{t \rightarrow+0}(\omega)^{*}(t)
$$

и, в частности, непрерьвность в нуле $(\omega)^{*}$, если $\omega$ непрерывна в нуле.

Исходя из функций $f$ и $u$, построим функции

$$
\begin{aligned}
& v_{x}^{+}(t)=\sup (\{0\} \cup\{f(y)-u(x): x y \leqslant t, y \in Y\}), \quad t \geqslant 0, \\
& v_{x}^{-}(t)=\sup (\{0\} \cup\{u(x)-f(y): x y \leqslant t, y \in Y\}), \quad t \geqslant 0 .
\end{aligned}
$$

Из теоремы 3.5 следует, что для любого $x \in X$ квазивогнутая функция $\omega_{x}^{+}=\left(v_{x}^{+}\right)^{*}$ обеспечивает выполнение условия (3.2), а квазивогнутая функция $\omega_{x}^{-}=\left(v_{x}^{-}\right)^{*}$ обеспечивает выполнение условия (3.3).

Из (3.7) следуют равенства

$$
\varlimsup_{x_{1} \rightarrow y} u\left(x_{1}\right)=\varlimsup_{y_{1} \rightarrow y} f\left(y_{1}\right), \quad \underline{\lim }_{x_{1} \rightarrow y} u\left(x_{1}\right)=\varliminf_{y_{1} \rightarrow y} f\left(y_{1}\right), \quad y \in Y,
$$

и, в частности, непрерывность МКЛ-продолжения в точках непрерывности исходной функции $f$.

Таким образом, с помощью МКЛ-продолжения получены положительные ответы на все вопросы, сформулированные в первом параграфе. 


\section{§4. Задача о следах функций с ограниченным градиентом}

В этом параграфе рассматривается задача, в которой использование МКЛ-продолжения вместо продолжения Макшейна позволяет усилить и обобщить результат.

ОПРЕДЕЛЕНИЕ. Нормой градиента функции $u: X \rightarrow \mathbb{R}$ в точке $x \in X$ будем называть величину

$$
\|D u(x)\|=\varlimsup_{z \rightarrow x} \frac{|u(z)-u(x)|}{x z} .
$$

Отметим, что в случае, когда $X=\mathbb{R}^{n}$ и $u$ дифференцируема, это определение дает обычную норму градиента. Однако в общем случае градиент не определен, а определенная вьшше норма градиента служит мерой скорости изменения значения функции в точке.

В [7] рассмотрены функции, удовлетворяющие условию $\|D u(x)\|<1$. В случае $X=\mathbb{R}^{n}$ такие функции называются функциями с пространственноподобными графиками и имеют физические приложения. Задача заключается в характеризации следов таких функций на множестве $Y$. В [7], [21] показано, что ее решение позволяет получить условия сушествования решения задачи Дирихле для уравнения максимальных поверхностей в пространстве Минковского. Сформулируем задачу в терминах продолжения функций:

найти необходимье и достаточнье ограничения на функцию $f: Y \rightarrow \mathbb{R}$, обеспечивающие существование продолжения и, непрерьвного на $X u$ удовлетворяющего условию

$$
\|D u(x)\|<1 \quad \forall x \in X \backslash Y .
$$

В этом параграфе будем предполагать выполнение следующих условий:

1) для любых двух точек $x_{1}, x_{2} \in X$ существует отображение $x:[0,1] \rightarrow X$ (называемое дугой) такое, что $x(0)=x_{1}, x(1)=x_{2}, x(t) \in X \backslash Y$ при $0<t<1$

2) $d\left(x_{1}, x_{2}\right)$ есть точная нижняя грань длин дуг, указанных в 1 ) (определение длины дуги см., например, в [22]).

Из этих условий следует линейная связность $X$ и $X \backslash Y$, а также отсутствие внутренних точек в $Y$. Будем использовать лемму, которая следует из основного результата работы [22] (см. также [17, лемма 5]).

ЛЕмма 4.1. При выполнении указанных условий, если $\|D u(x)\| \leqslant M$ для любого $x \in X$, mo

$$
\left|u\left(x_{1}\right)-u\left(x_{2}\right)\right| \leqslant M \cdot x_{1} x_{2} \quad \forall x_{1}, x_{2} \in X
$$

В общем случае получены не совпадающие между собой необходимые и достаточные условия существования непрерывного продолжения со свойством (4.1). 
ТЕОРема 4.1. Для существования непрерывного продолжения со свойством (4.1) необходимо, чтобы выполнялось следующее условие:

$a(x)=\sup \left\{f\left(y_{1}\right)-f\left(y_{2}\right)-\left(x y_{1}+x y_{2}\right): y_{1}, y_{2} \in Y\right\}<0 \quad \forall x \in X \backslash Y$.

ДоКАЗАТЕЛЬСтво. Пусть продолжение $u$ обладает свойством (4.1). Пусть $x \in X \backslash Y$. Из неравенства $\|D u(x)\|<1$ следует существование $\varepsilon>0$ и $r>0$ таких, что

$$
u(z)-u(x) \leqslant x z(1-2 \varepsilon) \quad \forall z \in B_{r}(x) \subset X \backslash Y,
$$

где $B_{r}(x)$ - замкнутый шар радиуса $r$ с центром в $x$. Возьмем произвольные $y_{1}, y_{2} \in Y$. Соединим точки $x$ и $y_{1}$ дугой $\gamma$, длина которой не превышает $x y_{1}+r \varepsilon$. Точку пересечения дуги и $\partial B_{r}(x)$ обозначим через $x_{1}$. Очевидно, что $x x_{1}+x_{1} y_{1} \leqslant x y_{1}+r \varepsilon$ и

$$
u\left(x_{1}\right)-u(x) \leqslant x x_{1}-2 r \varepsilon .
$$

Воспользовавшись леммой 4.1 , получаем

$$
f\left(y_{1}\right)-u\left(x_{1}\right) \leqslant y_{1} x_{1}, \quad u(x)-f\left(y_{2}\right) \leqslant x y_{2} .
$$

Складывая три последних неравенства, получаем

$$
f\left(y_{1}\right)-f\left(y_{2}\right) \leqslant y_{1} x_{1}+x_{1} x+x y_{2}-2 r \varepsilon \leqslant x y_{1}+x y_{2}-r \varepsilon
$$

откуда следует (4.2). Теорема доказана.

ТЕОРема 4.2. Для существования непрерывного продолжсения со свойством (4.1) достаточно, чтобь выполнялось следующее условие:

$$
L(x)=\sup \left\{\frac{f\left(y_{1}\right)-f\left(y_{2}\right)}{x y_{1}+x y_{2}}: y_{1}, y_{2} \in Y\right\}<1 \quad \forall x \in X \backslash Y .
$$

ДоКАЗАТЕЛЬСТво. При выполнения условия теоремы МКЛ-продолжение обладает свойством (4.1). Это следует из теоремы 3.2 и очевидного неравенства

$$
\|D u(x)\| \leqslant k[X] u(x)=L(x) .
$$

Теорема доказана.

СлЕДСТВИЕ 1. Если $Y$ ограничено, то для существования непрерывного продоләсения со свойством (4.1) необходимо и достаточно, чтобы выполнялось условие (4.2).

ДокАзАтЕльство. Требует доказательства только достаточность. Пусть $x \in X \backslash Y$. Из (4.2) следует, что для любых $y_{1}, y_{2} \in Y$

$$
\frac{f\left(y_{1}\right)-f\left(y_{2}\right)}{x y_{1}+x y_{2}}=\frac{f\left(y_{1}\right)-f\left(y_{2}\right)-\left(x y_{1}+x y_{2}\right)}{x y_{1}+x y_{2}}+1 \leqslant 1+\frac{a(x)}{\sup \{2 \cdot x y: y \in Y\}}<1 .
$$

Следовательно, $L(x)<1$, т.е. выполнено условие теоремы 4.2. Следствие доказано.

Из доказанного утверждения следует теорема 1 из [7]. При этом сняты условия локальной компактности пространства $(X, d)$ и компактности множества $Y$ и значительно сокращено доказательство. 
СЛЕДСТВИЕ 2. Если множество $Y$ неограничено, то для существования непрерывного продолжения со свойством (4.1) достаточно, чтобы выполнялось условие (4.2) и неравенства $p_{0}<1, q_{0}<1$, где $p_{0}$ и $q_{0}$ определень формулами (2.1) и (2.2).

ДокАЗАТЕЛьство. Из (4.2) следует, что $L(x) \leqslant 1 \forall x \in X \backslash Y$. Предположим, что $L(x)=1$. Рассмотрим $u=E_{k} f$. В соответствии с леммой 2.2 и следствием из нее функции $p_{x}(t), q_{x}(t)$ строго монотонны в некоторой окрестности точки $t=u(x)$ и сушествуют последовательности $\left\{y_{i}\right\}_{i=1}^{\infty}$ и $\left\{z_{i}\right\}_{i=1}^{\infty}$ точек из $Y$ такие, что

$$
u(x)=\lim _{i \rightarrow \infty}\left(f\left(y_{i}\right)+x y_{i}\right), \quad u(x)=\lim _{i \rightarrow \infty}\left(f\left(z_{i}\right)-x z_{i}\right) .
$$

Отсюда получаем

$$
\lim _{i \rightarrow \infty}\left(f\left(z_{i}\right)-f\left(y_{i}\right)-x y_{i}-x z_{i}\right)=0,
$$

что противоречит условию (4.2). Следовательно, $L(x)<1$, т.е. вьполнено условие теоремы 4.2. Следствие доказано.

Таким образом, между полученными необходимьми и достаточными условиями существования непрерывного продолжения со свойством (4.1) в случае неограниченного $Y$ имеется пробел. Есть основания предполагать, что условие (4.2) является не только необходимьм, но и достаточньм, однако пока это не доказано.

Рассмотрим случай, когда $Y$ неограничено и $\max \left\{p_{0} ; q_{0}\right\}=1$, не охваченный полученными результатами. В этом случае при выполнении (4.2) МКЛ-продолжение $u$ имеет вид:

если $p_{0}=1$ и $q_{0}<1$, то $u(x)=u_{-}(x)=\sup \{f(y)-x y: y \in Y\}$;

если $q_{0}=1$ и $p_{0}<1$, то $u(x)=u_{+}(x)=\inf \{f(y)+x y: y \in Y\}$;

если $q_{0}=1$ и $p_{0}=1$, то $u(x)=u_{0}(x)=\left(u_{-}(x)+u_{+}(x)\right) / 2$.

Это легко показывается с помощью леммы 2.6. Наиболее важна ситуация, когда $X$ - неограниченное подмножество $\mathbb{R}^{n}$. Она представляет интерес в связи с началом исследований уравнения максимальных поверхностей в неограниченных областях [23]. В этой ситуации при $\max \left\{p_{0} ; q_{0}\right\}=1$ продолжение со свойством (4.1) иногда реализуется в виде функции $u_{0}$. Например, когда функции $u_{-}, u_{+}$дифференцируемы и $D u_{-}(x) \neq D u_{+}(x)$ для любого $x \in X \backslash Y$.

ПримеР. Пусть из точки $O$ на плоскости исходят два луча $O A$ и $O B$ под углом $\alpha \geqslant \pi$. На лучах задана функция

$$
f(x)=\left\{\begin{aligned}
\beta \max \{0 ;(O x-1)\}, & \text { если } x \in O A, \\
-\beta \max \{0 ;(O x-1)\}, & \text { если } x \in O B .
\end{aligned}\right.
$$

Расстояние между двумя точками в пределах угла $A O B$ определяется как длина кратчайшей ломаной, лежащей в пределах угла и соединяющей эти точки. Определим значения параметров, при которых возможно непрерьвное продолжение функции $f$ на внутренность угла $A O B$ со свойством (4.1).

При $\beta>1$ не выполняется условие теоремы 4.1, следовательно, требуемого продолжения не существует. При $\beta<1$ в соответствии со следствием 2 теоремы 4.2 МКЛ-продолжение обладает требуемым свойством. При $\alpha>\pi, \beta=1$ легко видеть, что функция $u_{0}$ дает требуемое продолжение. Случай $\alpha=\pi, \beta=1$ полученными результатами не охватьвается. 


\section{§5. Применение продолжения функций в задачах приближения}

\section{1. Приближение посредством функций, обращающихся в нуль на $Y$.} Примем обозначения из работы [12]. Определим нормы на множестве ограниченных липшицевых функций:

$$
\begin{gathered}
\|h\|_{C}=\sup \{|h(x)|: x \in \operatorname{dom}(h)\}, \\
\|h\|_{s}=\|h\|_{C}+\|h\|_{\text {Lip }}, \quad\|h\|_{m}=\max \left\{\|h\|_{C} ;\|h\|_{\text {Lip }}\right\} .
\end{gathered}
$$

Через $Y^{\perp}$ обозначим множество всех функций, определенных на $X$ и обрашающихся в нуль на $Y$. На $\operatorname{Lip}(X) \cap Y^{\perp}$ липшицева полунорма является нормой.

В этом пункте рассматривается задача приближения функции $g: X \rightarrow \mathbb{R}$ посредством функций из $Y^{\perp}$ в нормах $\|\cdot\|_{\text {Lip }},\|\cdot\|_{s},\|\cdot\|_{m}$.

Введем функцию $f=g \mid Y$. Пусть $f \in \operatorname{Lip}(Y)$ и $u$-продолжение $f$ на $X$ такое, что $\|f\|_{\text {Lip }}=\|u\|_{\text {Lip. }}$ В этом случае, как показано в [12], [13], функция $g_{0}=g-u$ является наилучшим приближением функции $g$ в норме $\|\cdot\|_{\text {Lip }}$. Если, кроме того, функция $f$ ограничена и выполняется условие $\|f\|_{C}=\|u\|_{C}$, то функция $g_{0}$ является наилучшим приближением функции $g$ в нормах $\|\cdot\|_{s}$ и $\|\cdot\|_{m}$.

Если $\|f\|_{\text {Lip }}=+\infty$, то задача поиска наилучшего приближения в традиционной постановке теряет смысл, так как

$$
\left\|g-g_{1}\right\|_{\text {Lip }}=+\infty \quad \forall g_{1} \in Y^{\perp} .
$$

Однако оказьвается возможным построить наилучшее приближение в обобшенном смысле.

Возьмем число $M>0$. Определим множество

$$
Y_{M}=\{y \in Y: k[Y] f(y)>M\} .
$$

Возьмем произвольное подмножество $Z \subset X$ такое, что

$$
d_{Z}=\inf \left\{d\left(x, Y_{M}\right): x \in Z\right\}>0 .
$$

Исходя из очевидного равенства

$$
\|h\|_{\text {Lip }}=\sup \{k[X] h(x): x \in X\},
$$

построим вспомогательные модификации рассматриваемых норм:

$$
\begin{gathered}
\|h\|_{\text {Lip }, Z}=\sup \{k[X] h(x): x \in Z\}, \\
\|h\|_{s, Z}=\|h\|_{C}+\|h\|_{\text {Lip }, Z}, \quad\|h\|_{m, Z}=\max \left\{\|h\|_{C} ;\|h\|_{\text {Lip }, Z}\right\} .
\end{gathered}
$$

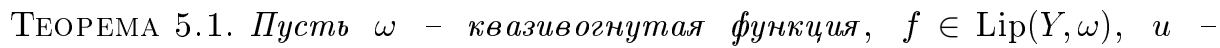
МКЛ-продолжение функции $f, Z \subset X$ и выполняется (5.1). Тогда функция $g_{0}=g-u$ является наилучшим приближением функции $g$ в норме $\|\cdot\|_{\operatorname{Lip}, Z}$. Если при указанных условиях функиия $f$ ограничена, то функция $g_{0}$ является наилучшим приближением функции $g$ в нормах $\|\cdot\|_{s, Z} u\|\cdot\|_{m, Z}$. 
ДоКАЗАТЕЛЬСТво. Пусть $x \in Z \backslash Y$. Из теоремы 3.2 следует

$$
k[X] u(x)=\sup \left\{\frac{f\left(y_{1}\right)-f\left(y_{2}\right)}{x y_{1}+x y_{2}}: y_{1}, y_{2} \in Y\right\}=\max \left\{A_{1} ; A_{2}\right\}
$$

где

$$
\begin{aligned}
& A_{1}=\sup \left\{\frac{f\left(y_{1}\right)-f\left(y_{2}\right)}{x y_{1}+x y_{2}}: y_{2} \in Y, y_{1} \in Y_{M}\right\} \\
& A_{2}=\sup \left\{\frac{f\left(y_{1}\right)-f\left(y_{2}\right)}{x y_{1}+x y_{2}}: y_{2} \in Y, y_{1} \in Y \backslash Y_{M}\right\} .
\end{aligned}
$$

Оценим $A_{1}$, воспользовавшись возрастанием функции $\omega$ и убьванием функции $\omega(t) / t$

$$
\begin{aligned}
A_{1} & \leqslant \sup \left\{\frac{\omega\left(y_{1} y_{2}\right)}{x y_{1}+x y_{2}}: y_{2} \in Y, y_{1} \in Y_{M}\right\} \\
& \leqslant \sup \left\{\frac{\omega\left(x y_{1}+x y_{2}\right)}{x y_{1}+x y_{2}}: y_{2} \in Y, y_{1} \in Y_{M}\right\} \leqslant \frac{\omega\left(d_{Z}\right)}{d_{Z}}<+\infty .
\end{aligned}
$$

Оценим $A_{2}$, воспользовавшись следствием теоремы 3.3 ,

$$
A_{2} \leqslant \sup \left\{\frac{f\left(y_{1}\right)-f\left(y_{2}\right)}{y_{1} y_{2}}: y_{2} \in Y, y_{1} \in Y \backslash Y_{M}, y_{1} \neq y_{2}\right\} \leqslant M
$$

В итоге имеем $k[X] u(x)<+\infty$.

Следствие теоремы 3.3 позволяет утверждать, что

$$
k[X] u(y)=k[Y] f(y) \leqslant M \quad \forall y \in Z \cap Y .
$$

Следовательно, $\|u\|_{\operatorname{Lip}, Z}<+\infty$. Так как отклонение функции $g$ от функции $g_{1} \in$ $Y^{\perp}$ является продолжением функции $f$, то из неравенства (3.1) следует

$$
k[X] g_{0}(x) \leqslant k[X]\left(g-g_{1}\right)(x) \quad \forall x \in Z .
$$

Отсюда получается требуемый результат для нормы $\|\cdot\|_{\text {Lip }, Z}$. Если функция $f$ ограничена, то из теоремы 3.1 следует, что $\|u\|_{C}=\|f\|_{C}$, откуда получается требуемый результат для норм $\|\cdot\|_{s, Z}$ и $\|\cdot\|_{m, Z}$. Теорема доказана.

Таким образом, показано, что наилучшее относительно вспомогательных норм приближение $g_{0}=g-u$ не зависит от выбора числа $M$ и множества $Z$. Поэтому его естественно принять за решение задачи наилучшего приближения в исходных нормах.

5.2. Плотность $\operatorname{Lip}(X)$ в $\operatorname{lip}(X, \omega)$. Пусть $\omega(t)>0$ при $t>0$. Определим $\operatorname{lip}(X, \omega)$ как класс всех функций $h$, определенных на $X$ и удовлетворяюших условию

$$
\lim _{t \rightarrow+0} \frac{\sup \left\{h\left(x_{1}\right)-h\left(x_{2}\right): x_{1} x_{2} \leqslant t\right\}}{\omega(t)}=0 .
$$

В [11] доказано следующее утверждение. 
ТеОРема 5.2. Пусть $(X, d)$ компактно, $\omega$ - непрерывная в нуле, квазивогнутая функиия $u \lim _{t \rightarrow+0} \omega(t) / t=+\infty$. Тогда класс $\operatorname{Lip}(X)$ плотен в $\operatorname{lip}(X, \omega)$, т.е. для любой функиии $g \in \operatorname{lip}(X, \omega)$ существует функиия $u \in \operatorname{Lip}(X)$ сколь угодно близкая к $g$ в норме

$$
\|h\|_{C, \omega}=\max \left\{\|h\|_{C} ;\|h\|_{\omega}\right\},
$$

$2 \partial e$

$$
\|h\|_{\omega}=\sup \left\{\frac{\left|h\left(x_{1}\right)-h\left(x_{2}\right)\right|}{\omega\left(x_{1} x_{2}\right)}: x_{1}, x_{2} \in X, x_{1} \neq x_{2}\right\} .
$$

Ранее этот результат был доказан в [24] для $\omega(t)=t^{\alpha}, 0<\alpha<1$. Доказательства в [11], [24] основаны на рассмотрении сопряженного к $\operatorname{lip}(X, \omega)$ пространства, которое является пространством мер. Используя МКЛ-продолжение, получим прямое доказательство теоремы, указываюшее способ построения приближаюшей функции.

ДокаЗАтЕльство. Пусть $g \in \operatorname{lip}(X, \omega)$. Определим возрастающую функцию

$$
\omega_{g}(t)=\sup \left\{\left|g\left(x_{1}\right)-g\left(x_{2}\right)\right|: x_{1} x_{2} \leqslant t\right\}, \quad t \geqslant 0 .
$$

Из определения класса $\operatorname{lip}(X, \omega)$ и непрерывности в нуле функции $\omega$ следует непрерывность функции $g$. Из компактности пространства $(X, d)$ следует ограниченность $g$. Поэтому функция $\omega_{g}$ ограничена. Построим в соответствии с теоремой 3.5 квазивогнутую мажоранту этой функции $\omega^{*}=\left(\omega_{g}\right)^{*}$.

Пусть $Y$ - конечная $\varepsilon$-сеть в $(X, d)$, т.е. конечное подмножество множества $X$ такое, что для любого $x \in X$ сушествует точка $y(x) \in Y$, удаленная от $x$ не более чем на $\varepsilon$. Пусть $f=g \mid Y, u$ - МКЛ-продолжение функции $f$ на $X$. Из конечности множества $Y$ следует, что $f \in \operatorname{Lip}(Y)$. Из теоремы 3.4 получаем, что $u \in \operatorname{Lip}(X)$ и $u \in \operatorname{Lip}\left(X, \omega^{*}\right)$.

Оценим близость функций $g$ и $u$. Пусть $h=g-u$. Тогда $h(y(x))=0$ для любого $x \in X$. Оценим сверху величину $\|h\|_{C}$. Справедливо следующее:

$$
\begin{aligned}
|h(x)|=|h(x)-h(y(x))| & \leqslant|g(x)-g(y(x))|+|u(x)-u(y(x))| \\
& \leqslant \omega_{g}(\varepsilon)+\omega^{*}(\varepsilon) \leqslant 2 \omega^{*}(\varepsilon) .
\end{aligned}
$$

Следовательно, $\|h\|_{C} \leqslant 2 \omega^{*}(\varepsilon)$ и $\|h\|_{C} \rightarrow 0$ при $\varepsilon \rightarrow 0$.

Справедливы неравенства

$$
\begin{aligned}
\left|h\left(x_{1}\right)-h\left(x_{2}\right)\right| & =\left|h\left(x_{1}\right)-h\left(y\left(x_{1}\right)\right)+h\left(y\left(x_{2}\right)\right)-h\left(x_{2}\right)\right| \\
& \leqslant\left|h\left(x_{1}\right)-h\left(y\left(x_{1}\right)\right)\right|+\left|h\left(y\left(x_{2}\right)\right)-h\left(x_{2}\right)\right| \leqslant 4 \omega^{*}(\varepsilon), \\
\left|h\left(x_{1}\right)-h\left(x_{2}\right)\right| & \leqslant\left|g\left(x_{1}\right)-g\left(x_{2}\right)\right|+\left|u\left(x_{1}\right)-u\left(x_{2}\right)\right| \\
& \leqslant \omega_{g}\left(x_{1} x_{2}\right)+\omega^{*}\left(x_{1} x_{2}\right) \leqslant 2 \omega^{*}\left(x_{1} x_{2}\right),
\end{aligned}
$$

из которых следует, что

$$
\left|h\left(x_{1}\right)-h\left(x_{2}\right)\right| \leqslant 4 \omega^{*}\left(\min \left\{\varepsilon ; x_{1} x_{2}\right\}\right) .
$$

В результате имеем

$$
\|h\|_{\omega} \leqslant \sup \left\{\frac{4 \omega^{*}\left(\min \left\{\varepsilon ; x_{1} x_{2}\right\}\right)}{\omega\left(x_{1} x_{2}\right)}: x_{1}, x_{2} \in X, x_{1} \neq x_{2}\right\} \leqslant \sup \left\{\frac{4 \omega^{*}(t)}{\omega(t)}: t \leqslant \varepsilon\right\} .
$$


Для завершения доказательства достаточно показать, что

$$
\lim _{t \rightarrow+0} \frac{\omega^{*}(t)}{\omega(t)}=0
$$

Если $\sup \left\{\omega_{g}(s) / s: s>0\right\}<+\infty$, то (5.2) следует из условия

$$
\lim _{t \rightarrow+0} \frac{\omega(t)}{t}=+\infty
$$

Рассмотрим случай $\sup \left\{\omega_{g}(s) / s: s>0\right\}=+\infty$. Из теоремы 3.5 следует, что для каждого достаточно малого $t>0$ сушествует число $s_{t} \geqslant t$ такое, что

$$
\frac{\omega^{*}(t)}{t}=\frac{\omega_{g}\left(s_{t}+0\right)}{s_{t}}, \quad \lim _{t \rightarrow+0} s_{t}=0 .
$$

Получим

$$
\frac{\omega^{*}(t)}{\omega(t)}=\frac{\omega^{*}(t)}{t} \frac{t}{\omega(t)}=\frac{\omega_{g}\left(s_{t}+0\right)}{s_{t}} \frac{t}{\omega(t)} \leqslant \frac{\omega_{g}\left(s_{t}+0\right)}{s_{t}} \frac{s_{t}}{\omega\left(s_{t}\right)}=\frac{\omega_{g}\left(s_{t}+0\right)}{\omega\left(s_{t}\right)} .
$$

Известно $[19$, гл. $2, \S 1]$, что квазивогнутая функция непрерывна при $t>0$. Поэтому $\omega\left(s_{t}\right)=\omega\left(s_{t}+0\right)$. Из определения класса $\operatorname{lip}(X, \omega)$ следует, что

$$
\lim _{t \rightarrow+0} \frac{\omega_{g}(t)}{\omega(t)}=0 .
$$

Поэтому из (5.3) следует (5.2). Теорема доказана.

\section{Список литературы}

1. Wells J. H., Williams L. R. Embeddings and Extensions in Analysis. Berlin: Springer-Verlag, 1975.

2. McShane E. Extension of range of function // Bull. Amer. Math. Soc. 1934. V. 40. № 12 . P. 837-842.

3. Czipcer J., Geher L. Extension of functions satisfying a Lipschitz condition // Acta Math. Hungar. 1955. V. 6. № 1-2. P. 213-220.

4. Калябин Г. А. О следах функций с мажорируемыми производньми // Матем. заметки. 1975. T. 18. № 4. C. 499-506.

5. Владимиров Ю.Н. О максимальных функциях Липшица, связанных с задачей оптимального перемещения массы // Оптимизация. 1988. № 44. С. 96-113.

6. Sherbert $D$. The structure of ideals and point derivations in Banach algebras of Lipschitz functions // Trans. Amer. Math. Soc. 1964. V. 111. № 2. P. 240-272.

7. Клячин А. А.,Миклюков В. М. Следы функций с пространственноподобньми графиками и задача о продолжении функций при ограничениях на градиент // Матем. сб. 1992. T. 183. № 7. C. $49-64$.

8. Федерер Г. Геометрическая теория меры. М.: Наука, 1987.

9. Экланд И., Темам Р. Выпуклый анализ и вариационные проблемы. М.: Мир, 1979.

10. Hiriart-Urruty J.-B. Extension of Lipschitz functions // J. Math. Anal. Appl. 1980. V. 77. № 2. P. 539-555.

11. Hanin L.G. Kantorovich-Rubinshtein norm and its application in the theory of Lipschitz spaces // Proc. Amer. Math. Soc. 1992. V. 115. №2. P. 345-352. 
12. Mustata $C$. Extension of Hölder functions and some related problems of best approximation // Preprint. "Babes-Bolyai" Univ., Fac. Math. Phys., Res. Semin. № 7, 1991. P. 71-86.

13. Mabizela $S$. The relationship between Lipschits extensions, best approximations, and continuous selections // Quaestiones Math. 1991. V. 14. №3. P. 261-268.

14. Moreau P., Ronse $C$. Generation of shading-off in images by extrapolation of Lipschitz functions // Graphical Models and Image Processing. 1996. V. 58. № 4. P. 314-333.

15. Le Gruyer E., Archer J. C. Stability and convergence of extension schemes to continuous functions in general metric spaces // SIAM J. Math. Anal. 1996. V. 27. № 1. P. 274-285.

16. Мильман В. А. Продолжение функций с сохранением модуля непрерьвности // Весці АН Беларусі. Сер. фіз.-мат. навук. 1996. № 4. С. 23-28.

17. Мильман B. A. Продолжение функций, сохраняющее модуль непрерывности // Матем. заметки. 1997. Т. 61. № 2. С. 236-245.

18. Бурбаки Н. Функции действительного переменного. М.: Наука, 1965.

19. Крейн С.Г., Петунин Ю.И., Семенов Е. М. Интерполяция линейных операторов. М.: Наука, 1978.

20. Тиман $A . \Phi$. Теория приближения функций действительного переменного. М.: Физматгиз, 1960.

21. Bartnik R., Simon $L$. Spacelike hypersurfaces with prescribed boundary values and mean curvature // Comm. Math. Phys. 1982. V. 87. № 1. P. 131-152.

22. Sleźak B. A mean value theorem in metric spaces // Constructive Theory of Functions. Sofia: Publ. House Bulgarian Acad. Sci., 1988. P. 47-49.

23. Клячин A. A. Разрешимость задачи Дирихле для уравнения максимальных поверхностей с особенностями в неограниченных областях // Докл. АН. 1995. Т. 342. № 2 . C. $162-164$.

24. Bade W. G., Curtis P. C., Dales H. G. Amenability and weak amenability for Beurling and Lipschitz algebras // Proc. London Math. Soc. (3). 1987. V. 55. № 2. P. 359-377.

Институт технической кибернетики НАН Беларуси

Поступила в редакцию

E-mail: milman@newman.basnet.minsk.by

19.05.1997 